Check for updates

Cite this: RSC Adv., 2017, 7, 36392

\title{
Quercetin as a simple but versatile primer in dentin bonding†
}

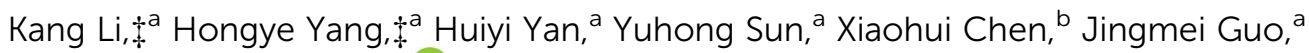 \\ Jiaxi Yue and Cui Huang (D) *a
}

Poor bonding stability remains the "Achilles' heel" of dentin bonding, a simple and effective approach to inhibit the activities of both exogenous collagenases and endogenous MMPs, and prevention of the growth of bacterial biofilm is therefore highly demanded. In the present study, a quercetin/ethanol solution was developed as a multifunctional primer to pretreat the dentin surface during adhesive restoration. The results showed that pretreatment with quercetin/ethanol solutions $(0.5$ and 1.0 $w t \%)$ on the dentin surface effectively preserved the bonding strength after one-month of collagenase aging $(p<0.05)$. Irrespective of aging, lower nanoleakage expression and less cohesive failure in dentin were observed in the quercetin-treated groups $(p<0.05)$. Compared with the control group, the in situ zymography test revealed that the 0.5 and $1.0 \mathrm{wt} \%$ quercetin-treated dentin effectively inhibited MMP activity, while their contact angles significantly increased. Live/dead bacterial staining and the MTT assay demonstrated that the 0.5 and $1.0 \mathrm{wt} \%$ quercetin groups exerted significant bactericidal effects while inhibiting biofilm growth of Streptococcus mutans ( $p<$ 0.05). We believe that a quercetin/ethanol solution might serve as a simple but versatile primer to provide dentists with a promising approach to obtain desirable bonding stability and to prevent secondary caries, thereby preventing the frequent replacement of resin-based restorations.

Received 6th July 2017

Accepted 15th July 2017

DOI: 10.1039/c7ra07467k

rsc.li/rsc-advances degradation occurs, the newly formed water-filled canals will allow dentinal fluids and bacteria to penetrate through the hybrid layer. ${ }^{6}$ Consequently, failure of resin-based restorations and secondary caries can occur.

To solve this problem, several strategies have been proposed, including ethanol-wet bonding, use of MMP inhibitors or collagen cross-linkers, and remineralization of the hybrid layer. ${ }^{7,8}$ Among these strategies, collagen crosslinkers have attracted great attention because of their clinical feasibility. Due to increasing concern about the cytotoxicity of chemical synthetics (glutaraldehyde (GA), carbodiimide, etc.), naturally derived materials, such as genipin, epigallocatechin-3-gallate (EGCG) and proanthocyanidins (PA), have become popular alternatives. ${ }^{9-12}$ Among the natural cross-linkers, genipin has much weaker crosslinking strength with protein and a much slower rate of cross-linking induction. ${ }^{12}$ Regarding EGCG, its high activity performance, which leads to discoloration, should not be overlooked for aesthetic materials. ${ }^{13}$ Although PA has an overall performance in dentin bonding, the antibacterial ability of PA against Streptococcus mutans (S. mutans) remains controversial, and its dark brown color might cause an aesthetic issue. ${ }^{14-19}$ Therefore, a simple and versatile material to preserve the integrity of hybrid layer, inhibit the activities of both exogenous collagenase and endogenous MMPs, and prevent the growth of bacterial biofilm is highly demanded. 


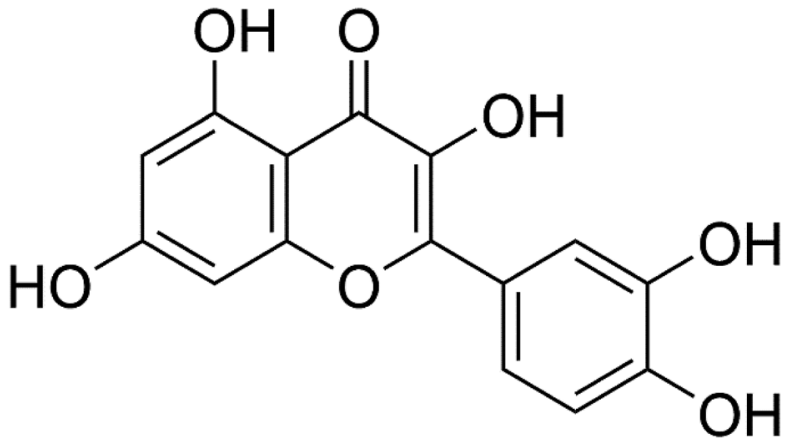

Fig. 1 Chemical structure of quercetin.

Quercetin (Fig. 1), a compound present in onions, tea, apples and so on, constitutes more than $70 \%$ of flavonoids. ${ }^{20}$ It has been documented that quercetin offers several benefits, including anti-inflammatory, antioxidant and cancerpreventing properties. ${ }^{21-23}$ Quercetin could inhibit the activity of MMP-2 and MMP-9 in PC-3 cells. ${ }^{24}$ Zhai and coworkers found that the cytotoxic concentration of quercetin is 100 times lower than that of GA, whereas the cross-linking effect on collagen was stronger than that of GA. ${ }^{25}$ In addition, quercetin exerts significant antimicrobial ability against various Gram-positive bacteria, Gram-negative bacteria, and viruses. $^{26,27}$ Furthermore, quercetin has a similar color to conventional adhesives, which might indicate less interference with aesthetic performance. Therefore, quercetin could be a promising additive for dentin bonding and could have the potential in preventing dental caries. However, to the best of our knowledge, no related reports have been available in the published literature until now.

This study aimed to explore the ability of quercetin/ethanol solution to serve as a therapeutic primer in dentin bonding via the evaluation of the physicochemical properties and antibacterial potential of adhesive/dentin interfaces. The null hypotheses are: quercetin pretreatment (1) does not affect the resin-dentin bond strength and interfacial nanoleakage expression after $24 \mathrm{~h}$ of water storage and one-month collagenase aging; (2) does not inhibit MMP activity in the hybrid layer; and (3) does not kill Streptococcus mutans or inhibit biofilm growth.

\section{Materials and methods}

\subsection{Preparation of experimental materials}

Since quercetin is barely soluble in water $\left(C_{\mathrm{q}}=60 \mathrm{mg} \mathrm{kg}^{-1}\right.$ at 25 ${ }^{\circ} \mathrm{C}$ ), pure ethanol was selected as the solvent $\left(C_{\mathrm{q}}=9.5 \mathrm{~g} \mathrm{~kg}^{-1}\right.$ at $25{ }^{\circ} \mathrm{C}$ ) in our experiments. ${ }^{28}$ Quercetin powder (Sigma-Aldrich, St. Louis, MO, USA) was directly added to $100 \%$ ethanol and water-bath heating at $37{ }^{\circ} \mathrm{C}$ for $15 \mathrm{~min}$ to prepare four experimental primer solutions at concentrations of $0,0.1,0.5$ and 1.0 $\mathrm{wt} \%$, respectively. The four primer solutions were designated as the following 4 groups and stored at $4{ }^{\circ} \mathrm{C}$ for further investigation:

Group 1: $100 \%$ ethanol (control group);
Group 2: 0.1 wt\% quercetin-containing ethanol (0.1 wt $\%$ group);

Group 3: $0.5 \mathrm{wt} \%$ quercetin-containing ethanol (0.5 wt $\%$ group);

Group 4: $1.0 \mathrm{wt} \%$ quercetin-containing ethanol (1.0 wt $\%$ group).

The collagenase solution was prepared by adding bacterial (Clostridium histolyticum) collagenase (Sigma-Aldrich, St. Louis, MO, USA) to artificial saliva to obtain a concentration of $0.1 \mathrm{mg}$ $\mathrm{mL}^{-1} .{ }^{29}$ The solution was stored at $-20{ }^{\circ} \mathrm{C}$ before use.

Fifty-two freshly extracted, intact human third molars were used. All teeth were collected after obtaining donors' informed consents, the protocol utilized in the present study [no. 2011(067)] was reviewed and approved by the Ethics Committee for Human Studies of the School and Hospital of Stomatology, Wuhan University, China. The methods employed were performed in accordance with the approved guidelines and regulations. All the teeth were stored in a $0.5 \%$ chloramine $\mathrm{T}$ solution at $4{ }^{\circ} \mathrm{C}$ for no more than one month.

\subsection{Microtensile bond strength (MTBS) test}

Twenty-four human third molars were employed for MTBS test. The teeth were sectioned parallel to the occlusal crown with a low-speed diamond saw (Isomet 11-1280-250; Buehler, Evanston, IL, USA) under water irrigation to expose the mid-coronal dentin. The flat dentin surface was wet-ground with 600-grit SiC paper for $60 \mathrm{~s}$ to create a uniform smear layer. The dentin surface of each specimen was etched with $35 \%$ phosphoric-acid gel (3M ESPE, St. Paul, MN, USA) for $15 \mathrm{~s}$ and rinsed with deionized water thoroughly. The specimens were randomly divided into four groups $(n=6)$, and pretreated using a microbrush for $60 \mathrm{~s}$ with 0 (control), 0.1, 0.5, or $1.0 \mathrm{wt} \%$ quercetin/ ethanol agents, respectively. After gentle drying with a filter paper, the specimens were bonded with Adper Single Bond 2 (3M ESPE, St. Paul, MN, USA), following the manufacturer's instructions. The adhesive was polymerized with a light-curing unit (Bluephase Style, Ivoclar-Vivadent Amherst, NY, USA) for 15 s. Resin composite (Charisma, Haraeus Kulzer, Hanau, Germany) build-ups with a total thickness of $4 \mathrm{~mm}$ were polymerized at $1 \mathrm{~mm}$ intervals with $20 \mathrm{~s}$ light curing.

After storage in deionized water at $37^{\circ} \mathrm{C}$ for $24 \mathrm{~h}$, the bonded teeth were sectioned parallel to the long axis to obtain slabs of $0.9 \mathrm{~mm}$ thickness. Four middle slabs from each group were stored for interfacial nanoleakage evaluation, and other slabs were further sectioned to produce beams of $0.9 \mathrm{~mm} \times 0.9 \mathrm{~mm}$. Ten qualified beams from each tooth were selected. Five beams ( $n=30$ total) were immediately subjected to MTBS test, and the other five ( $n=30$ total) were tested after one-month collagenase aging in the prepared $0.1 \mathrm{mg} \mathrm{mL}^{-1}$ collagenase-containing artificial saliva in the dark at $37{ }^{\circ} \mathrm{C}$ (the collagenase solution was replaced every 3 days).

Each beam used for MTBS was attached to a MTBS tester (Bisco Inc., Schaumburg, IL, USA) with cyanoacrylate glue (Zapit, Dental Ventures of America, Corona, CA, USA), and was loaded until failure at a cross-head speed of $1 \mathrm{~mm} \mathrm{~min}^{-1}$. The maximum failure load was recorded, the cross-sectional area of 
each beam was measured by digital calipers, and then the MTBS values (MPa) were calculated.

\subsection{Analysis of failure mode}

After MTBS test, the fractured dentin surfaces were mounted, dehydrated, sputter-coated with gold, and then observed via field-emission scanning electron microscopy (FESEM) (Sigma, Zeiss, Germany). The failure modes were classified into four groups: $(A)$ adhesive failure; (CD) cohesive failure in dentin; (CC) cohesive failure in composite; and $(M)$ mixed failure. ${ }^{30}$

\subsection{Interfacial nanoleakage evaluation}

The four stored middle slabs from each group were randomly assigned to be treated immediately or after one-month collagenase aging ( $n=2$ each subgroup). Slab surfaces were coated with two layers of nail varnish, leaving a blank $1 \mathrm{~mm}$-width area along the bonded interface. The specimens were placed in 50 $\mathrm{wt} \%$ ammoniac $\mathrm{AgNO}_{3}$ solution in the dark for $24 \mathrm{~h}$, rinsed with deionized water thoroughly, and then immersed in a photodeveloping solution for $8 \mathrm{~h}$ under fluorescent light irradiation. The specimens were wet-polished with 600-, 1200-, 2000-, and 5000-grit $\mathrm{SiC}$ abrasive papers and diamond paste $(0.5 \mu \mathrm{m}$, Lab Testing Technology, Shanghai, China), cleaned ultrasonically, dried and sputter-coated with carbon. Ten randomly captured fields along the dentin-bonding interface of each slab (20 images for each subgroup) were recorded under FESEM. Image J software (NIH, Frederick, MD, USA) was utilized to calculate the nanoleakage percentage of silver nitrate deposition in the adhesive layer. The percentage was scored on a scale of $0-4$ in accordance with the classification developed by Sobia et al. as follows: 0 , no nanoleakage; $1,<25 \%$ nanoleakage; 2 , $25 \% \leq 50 \%$ nanoleakage; $3,50 \% \leq 75 \%$ nanoleakage; and 4 , $>75 \%$ nanoleakage. ${ }^{31}$

\subsection{Surface contact angle test}

Sixteen human third molars were sectioned parallel to the occlusal surface to remove the enamel crown, and two dentin disks of $0.5 \mathrm{~mm}$ thickness were obtained from each tooth. The dentin disks were randomly assigned to four experimental groups ( $n=8$ disks per group). The disks were wet-polished with 600-, 1200- and 2000-grit $\mathrm{SiC}$ abrasive papers and diamond paste $(0.5 \mu \mathrm{m})$, then cleaned ultrasonically. For each experimental group, eight disks were etched with $35 \%$ phosphoric acid for $15 \mathrm{~s}$ and rinsed thoroughly, followed by pretreatment with microbrush for $60 \mathrm{~s}$ with 0 (control), 0.1, 0.5, or $1.0 \mathrm{wt} \%$ quercetin/ethanol solutions, respectively. Contact angles were measured with one drop $(5 \mu \mathrm{L})$ of deionized water on the dentin surface using a Dataphysics OCA 20 instrument (Dataphysics, Germany) at room temperature. The distance between the tip and the dentin surface was kept constant for all specimens.

\subsection{In situ zymography of the hybrid layer}

The quenched fluorescein-conjugated gelatin mixture (E-12055, Molecular Probes, Eugene, OR, USA) used for in situ zymography was prepared following the manufacturer's instructions and was stored at $-20^{\circ} \mathrm{C}$ until usage. Four human third molars were assigned to four groups, treated similar as described in Section 2.2. The bonded teeth were sectioned along the long axis to obtain slabs of $0.5 \mathrm{~mm}$ thickness to expose the adhesive-dentin interface. The slabs were wet-polished, ultrasonically cleaned and placed on glass slides. Fifty microliters of quenched fluorescein-conjugated gelatin mixture were dropped on top of each slab. The slabs were then covered by coverslips, transferred to humidified chambers and stored in $37^{\circ} \mathrm{C}$ for $24 \mathrm{~h}$ with light protection. The amount of green fluorescence produced by the dissolved quenched fluorescein-conjugated gelatin mixture by MMP was observed by confocal laser scanning microscopy (CLSM) (Fluoview FV1200, Olympus, Tokyo, Japan) with excitation of $488 \mathrm{~nm}$ and emission of lp530 nm.

\subsection{Bacterial culture and biofilm preparation}

S. mutans Ingbritt, obtained from the School of Stomatology, Wuhan University, was cultured in Brain Heart Infusion (BHI) broth (BD, Sparks, MD, USA) anaerobically at $37^{\circ} \mathrm{C}$ for $24 \mathrm{~h}$ and then adjusted to a concentration of approximately $10^{8} \mathrm{CFU}$ $\mathrm{mL}^{-1}$ for further usage.

Eight human third molars were sectioned following the same procedure described in Section 2.5 to yield 16 smooth dentin disks. The disks were disinfected under ultraviolet light for $1 \mathrm{~h}$ on each surface. The disks were randomly assigned to four groups $(n=4)$ and transferred to a 24-well plate. Four experimental solutions $(0,0.1,0.5$ or $1.0 \mathrm{wt} \%$ quercetin/ethanol) were applied on the top surface of each specimen for $60 \mathrm{~s}$ with a microbrush. A mixture of $10 \mu \mathrm{L}$ S. mutans cell suspension $\left(10^{8}\right.$ $\mathrm{CFU} \mathrm{mL}{ }^{-1}$ ) and $1 \mathrm{~mL}$ BHI added with $1 \%$ sucrose was injected into each well. After $24 \mathrm{~h}$ incubation at $37^{\circ} \mathrm{C}$ under anaerobic conditions, the biofilm-coated specimens were gently immersed in $1 \mathrm{~mL}$ of sterile phosphate buffer solution (PBS) twice to wash away the non-adherent bacteria cells. The dentin disks were then transferred to another 24-well plate with the top surface facing upward.

\subsection{Live/dead bacterial staining}

One biofilm-coated specimen from each group was stained with a live/dead bacterial viability kit (Molecular Probes, Invitrogen, USA) for $15 \mathrm{~min}$. The live bacteria could be stained by SYTO-9 to emit green fluorescence, whereas dead bacteria with damaged membrane could be penetrated by propidium iodide (PI) to emit red fluorescence. ${ }^{32}$ The excitation wavelengths for SYTO-9 and PI were 488 and $568 \mathrm{~nm}$ respectively. The specimens were observed by CLSM at $40 \times$ magnification. Two representative image stacks (Z-stack) of the biofilm were obtained for each specimen at a Z-step of $2 \mu \mathrm{m}$ (i.e., distance between two consecutive images in a stack), starting from the bottom of the biofilm that was in contact with the pretreatment surface, to the top of the biofilm. BioImageL software (Faculty of Odontology, Malmö University, Malmö, Sweden) was used to analyze the confocal images from each Z-stack. The total biomass and live/ dead bacterial distributions from the first 10 layers of the Zstack (i.e. 1st-10th images, in total $20 \mu \mathrm{m}$ thickness) were 
analyzed to evaluate the antibacterial effect of quercetin-treated dentin surfaces.

\subsection{Antibacterial evaluation by MTT assay}

Three biofilm-coated specimens from each group were transferred to a 12-well plate and each well was added with $1 \mathrm{~mL}$ of 3(4,5-dimethylthiazol-2-yl)-2,5-diphenyltetrazolium bromide (MTT; Sigma Chemical Co., St. Louis, USA) solution $(0.5 \mathrm{mg}$ $\mathrm{mL}^{-1}$ ). After $1 \mathrm{~h}$ of incubation at $37^{\circ} \mathrm{C}$ under an anaerobic environment, the MTT was converted to insoluble purple formazan crystals because of the dehydrogenase activity of the live bacteria. ${ }^{33}$ The MTT solution from each well was pipetted out and replaced with $1 \mathrm{~mL}$ of dimethyl sulfoxide (DMSO). After 10 min of gentle shaking, the supernatant was measured at $570 \mathrm{~nm}$ using a spectrophotometer (Powerwave 340, Bio-tek Instruments, Winooski, VT, USA). Because quercetin is known to have intrinsic fluorescence and could be dissolved in DMSO, the background absorbance of each quercetin concentration was measured separately, and was subtracted from the $\mathrm{OD}_{570}$ value. $^{34}$

\subsection{Cytotoxicity evaluation by MTT assay}

Human dental pulp cells (HDPs) were cultured in $\alpha$-modified essential medium (HyClone, Logan, UT, USA) supplemented with $10 \%$ fetal bovine serum at $37{ }^{\circ} \mathrm{C}$ with $5 \% \mathrm{CO}_{2}$ in a humidified environment. The HDPs were chosen from third passage. Due to the limited solubility of quercetin in ethanol, different amounts of quercetin were added to DMSO solution to yield a series of increasing concentrations of quercetin/ DMSO solutions $\left(0,1.25,2.5,5,10,20,40 \mathrm{mg} \mathrm{mL}^{-1}\right)$. The prepared solutions were added to $\alpha$-modified essential medium at a ratio of $1: 99$ to yield quercetin-containing medium $\left(0,12.5,25,50,100,200,400 \mu \mathrm{g} \mathrm{mL}^{-1}\right)$. HDPs were added to a 96-well plate with density of 5000 cells per well and cultured at $37^{\circ} \mathrm{C}$ with $5 \% \mathrm{CO}_{2}$ for $24 \mathrm{~h}$. Then, the old medium was pipetted out, with a series of different quercetincontaining media added. After $24 \mathrm{~h}$ culturing, each well had $10 \mu \mathrm{L}$ of MTT solution $\left(5 \mathrm{mg} \mathrm{mL}^{-1}\right)$ added and incubated for $4 \mathrm{~h}$. Then the quercetin-containing medium was removed, and $150 \mu \mathrm{L}$ of DMSO solution was added to each well. The optical density of each well was measured following the same procedure described in Section 2.9, and the background absorbance was subtracted. The results were expressed as the relative cell viability (\%) compared with the control group (1\% DMSO-containing medium). The experiment was performed in quintuplicate.

\subsection{Statistical analysis}

The MTBS test results were analysed using two-way (variables: different quercetin concentrations and collagenase aging) analysis of variance (ANOVA) and post hoc Tukey's test, after validating the equal variance assumptions of the data. Statistical differences between the scores of the nanoleakage groups were analysed using the Kruskal-Wallis test with Dunnett's post hoc test while inter-examiner reliability was assessed using the Cohen's kappa test. After equal variance assumptions confirmed, contact angles, live bacteria distributions, and MTT assay results were analyzed using one-way analysis of variance (ANOVA) followed by post hoc Tukey's test. All statistical analyses were performed using SPSS (IBM SPSS Statistics 20, Armonk, NY, USA). The significance level was set at 0.05 for all tests.

\section{Results}

\subsection{MTBS}

The MTBS data obtained from all the groups are shown in Table 1. In the immediate groups, no significant difference on bond strength was found between different pretreatment solutions ( $p$ $>0.05)$. However, in the collagenase-aging groups, the 0.5 and $1.0 \mathrm{wt} \%$ groups showed significantly higher $(p<0.05)$ bond strengths than the other two groups. Furthermore, one-month collagenase aging did not affect the bond strengths of the 0.5 and $1.0 \mathrm{wt} \%$ quercetin-pretreated groups. Two-way ANOVA showed that the variables of pretreatment solution $(F=10.675$, $p=0.000)$ and collagenase aging $(F=52.154, p=0.000)$ significantly affected bond strength. The interaction of pretreatment solution $\times$ collagenase aging was also significant $(F=11.934, p=0.000)$, indicating that the changes in MTBS were dependent on these two factors.

\subsection{Failure mode analysis}

After the MTBS test, the frequency distribution of failure modes is presented in Table 1 . In the immediate groups, the dominant failure mode in the 0.5 and $1.0 \mathrm{wt} \%$ quercetinpretreated groups was adhesive failure, whereas cohesive failure in dentin increased in the other two groups. After onemonth collagenase aging, the frequency of mixed failure increased in all groups. Fig. 2 shows the representative FESEM images of the failure modes from the immediate and aged groups. A cohesive failure in the dentin was noted in specimens from the immediate control group (Fig. 2A). The failure was mainly found at the bottom of the hybrid layer,

Table 1 Microtensile bond strength (MTBS) and failure frequency of each group ${ }^{a}$

\begin{tabular}{lllllll}
\hline & & & \multicolumn{3}{c}{ Failure frequency (\%) } \\
\cline { 5 - 7 } Groups & Aging & MTBS (MPa) & $A$ & CC & CD & $M$ \\
\hline \multirow{2}{*}{ Control } & Immediate & $37.84 \pm 6.69^{\mathrm{a}}$ & 35 & 10 & 25 & 30 \\
& Aged & $26.18 \pm 6.27^{\mathrm{b}}$ & 25 & 5 & 15 & 55 \\
\multirow{2}{*}{$0.1 \mathrm{wt} \%$} & Immediate & $38.65 \pm 5.23^{\mathrm{a}}$ & 35 & 10 & 15 & 40 \\
& Aged & $27.58 \pm 5.91^{\mathrm{b}}$ & 25 & 15 & 10 & 50 \\
\multirow{2}{*}{$0.5 \mathrm{wt} \%$} & Immediate & $38.09 \pm 6.78^{\mathrm{a}}$ & 60 & 20 & 0 & 20 \\
& Aged & $36.71 \pm 7.86^{\mathrm{a}}$ & 55 & 10 & 0 & 35 \\
\multirow{2}{*}{$1.0 \mathrm{wt} \%$} & Immediate & $37.73 \pm 7.02^{\mathrm{a}}$ & 70 & 10 & 0 & 20 \\
& Aged & $36.95 \pm 7.24^{\mathrm{a}}$ & 55 & 5 & 0 & 40
\end{tabular}

${ }^{a}$ The MTBS values are expressed as means \pm standard deviation. Groups with the same letters are not statistically different $(p>0.05)$. $A$, adhesive failure; CC, cohesive failure in composite; $\mathrm{CD}$, cohesive failure in dentin; $M$, mixed failure. 

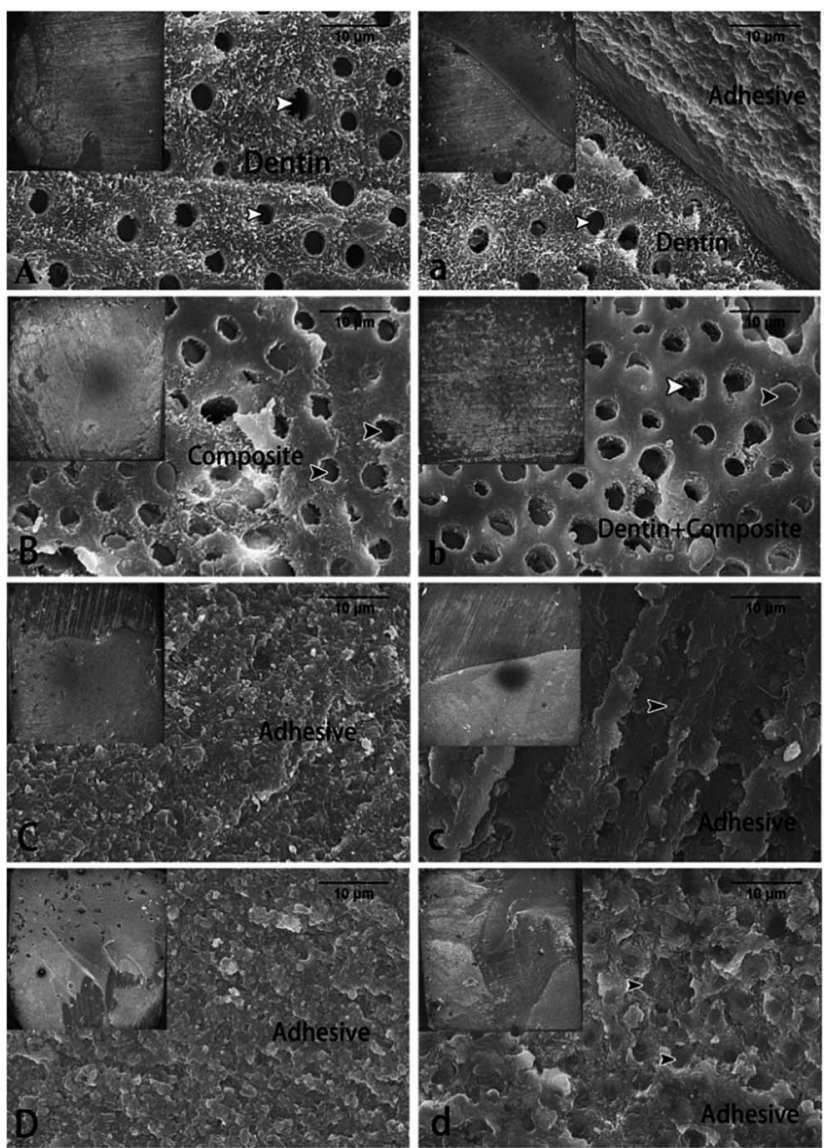

Fig. 2 Representative FESEM images (high magnification, 2000x) of the four experimental groups at immediate testing (left column) and after one-month collagenase aging (right column). Low magnification images at a magnification of $80 \times$ presented at the upper left part illustrated the general condition of the fracture surfaces. (A) Cohesive failure in dentine; (B) cohesive failure in composite; (C) adhesive failure; (D) adhesive failure. (a) Cohesive failure in dentin and adhesive failure. (b) Cohesive failure in dentin and cohesive failure in composite. (c) and (d) Adhesive failure, some resin tags were observed under the adhesive layer. Black arrow: resin tags; white arrow: open dentin tubules.

and the dentin tubules were observed. Specimens from the immediate $0.1 \mathrm{wt} \%$ group demonstrated cohesive failure in the composite (Fig. 2B), and the resin tags confirmed that failure occurred at the top of the hybrid layer. In the immediate 0.5 and $1.0 \mathrm{wt} \%$ groups, adhesive failure was observed (Fig. 2C and D), and the majority of the dentin surface was covered by adhesive resin. After one-month collagenase aging, the percentage of mixed failure increased in all groups. Unlike the immediate fractured surfaces, some resin tags were observed under the adhesive layer in the aged 0.5 and $1.0 \mathrm{wt} \%$ groups (Fig. $2 \mathrm{c}$ and d).

\subsection{Nanoleakage evaluation}

Statistical analysis results of nanoleakage expression are shown in Table 2. The inter-examiner reliability valued by Cohen's kappa test was $87 \%$. The results from the Kruskal-Wallis test showed that the specimens pretreated with 0.5 and $1.0 \mathrm{wt} \%$
Table 2 Percentage distribution of nanoleakage scores among the tested groups for immediate and after one-month collagenase aging ${ }^{a}$

\begin{tabular}{|c|c|c|c|c|}
\hline \multirow[b]{2}{*}{ Groups } & \multirow[b]{2}{*}{ Aging } & \multicolumn{3}{|c|}{ Nanoleakage expression } \\
\hline & & Score $0-4$ & $\%$ & Statistical difference \\
\hline \multirow[t]{10}{*}{ Control } & Immediate & 0 & 0 & A \\
\hline & & 1 & 0 & \\
\hline & & 2 & 30 & \\
\hline & & 3 & 35 & \\
\hline & & 4 & 35 & \\
\hline & Aged & 0 & 0 & $\mathrm{~A}$ \\
\hline & & 1 & 0 & \\
\hline & & 2 & 30 & \\
\hline & & 3 & 30 & \\
\hline & & 4 & 40 & \\
\hline \multirow[t]{10}{*}{$0.1 \mathrm{wt} \%$} & Immediate & 0 & 0 & A, B \\
\hline & & 1 & 30 & \\
\hline & & 2 & 30 & \\
\hline & & 3 & 30 & \\
\hline & & 4 & 10 & \\
\hline & Aged & 0 & 0 & $\mathrm{~B}, \mathrm{C}$ \\
\hline & & 1 & 50 & \\
\hline & & 2 & 45 & \\
\hline & & 3 & 5 & \\
\hline & & 4 & 0 & \\
\hline \multirow[t]{10}{*}{$0.5 \mathrm{wt} \%$} & Immediate & 0 & 30 & $\mathrm{~B}, \mathrm{C}$ \\
\hline & & 1 & 35 & \\
\hline & & 2 & 15 & \\
\hline & & 3 & 20 & \\
\hline & & 4 & 0 & \\
\hline & Aged & 0 & 10 & $\mathrm{~B}, \mathrm{C}$ \\
\hline & & 1 & 60 & \\
\hline & & 2 & 30 & \\
\hline & & 3 & 0 & \\
\hline & & 4 & 0 & \\
\hline \multirow[t]{10}{*}{$1.0 \mathrm{wt} \%$} & Immediate & 0 & 20 & $\mathrm{C}$ \\
\hline & & 1 & 70 & \\
\hline & & 2 & 0 & \\
\hline & & 3 & 10 & \\
\hline & & 4 & 0 & \\
\hline & Aged & 0 & 10 & $\mathrm{~B}, \mathrm{C}$ \\
\hline & & 1 & 80 & \\
\hline & & 2 & 10 & \\
\hline & & 3 & 0 & \\
\hline & & 4 & 0 & \\
\hline
\end{tabular}

${ }^{a}$ The Kruskal-Wallis test with Dunnett's post hoc test. Groups with the same letters are not statistically different $(p>0.05)$.

quercetin obtained significantly lower nanoleakage than the control group, irrespective of collagenase aging $(p<0.05)$. Representative FESEM images of nanoleakage expression are shown in Fig. 3. The bonded interface of the immediate control group showed extensive silver deposits throughout the hybrid layer (Fig. 3A). In comparison, the $0.1 \mathrm{wt} \%$ immediate group (Fig. 3B) demonstrated similar amounts of silver deposits as the immediate control group (Fig. 3A). Decreasing amounts of silver deposition in the hybrid layer were observed for the $0.5 \mathrm{wt} \%$ and $1.0 \mathrm{wt} \%$ groups (Fig. 3C and D). After one-month aging, several areas of the silver deposition in the control group demonstrated "water trees" morphologies which was similar to what was described by Tay (Fig. 3E and F). ${ }^{35}$ No signs of "water trees" were 

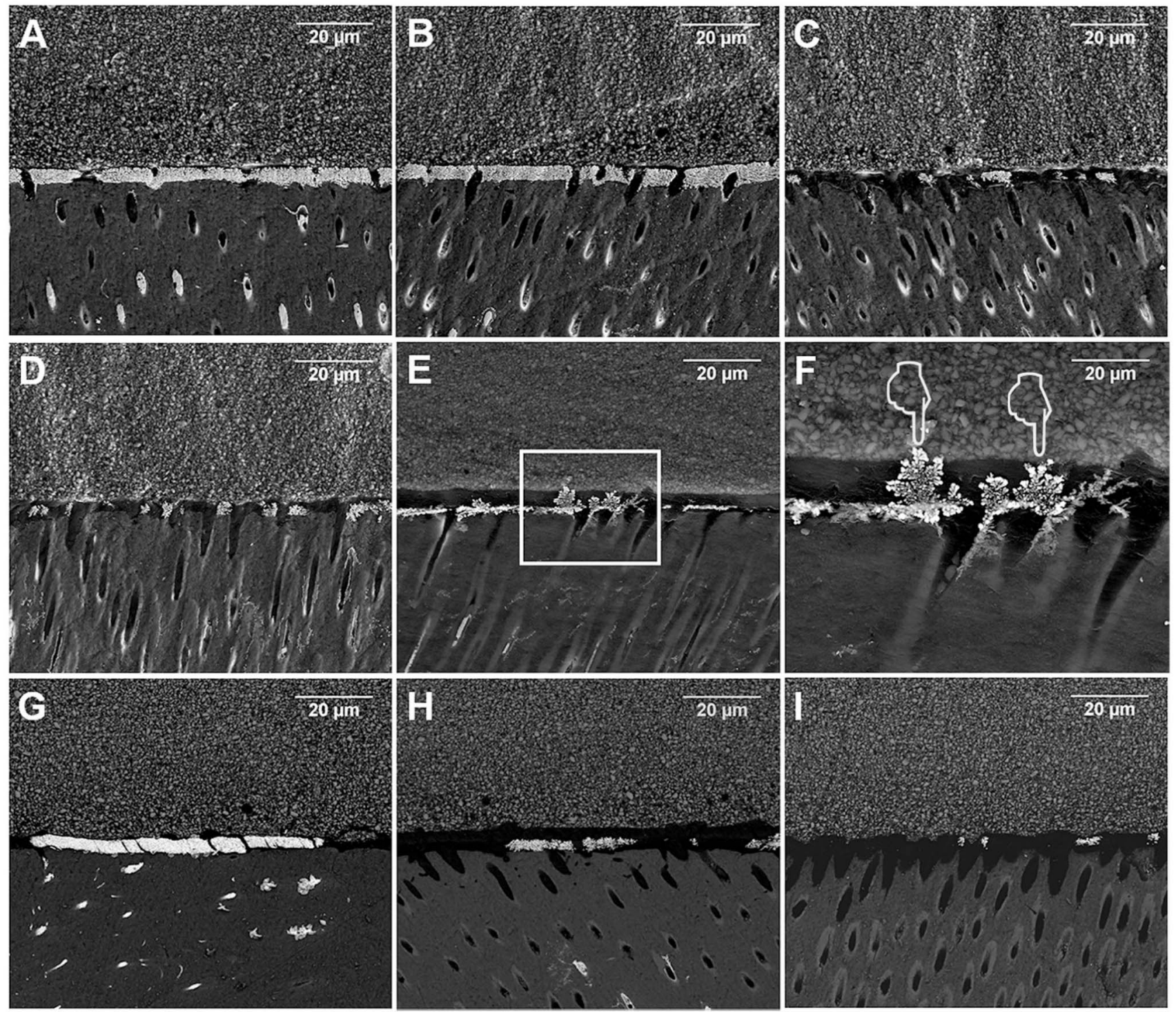

Fig. 3 Representative FESEM images $(3000 \times)$ of interfacial nanoleakage evaluation. Bonded interface of the immediate groups ((A) control; (B) $0.1 \mathrm{wt} \%$; (C) $0.5 \mathrm{wt} \%$; (D) $1.0 \mathrm{wt} \%$ ). Bonded interface of one-month collagenase aged groups ((E), control; (G) $0.1 \mathrm{wt} \%$; (H) 0.5 wt\%; (I) 1.0 wt\%). The high-magnification image of (E) is shown in (F) $(8000 \times)$, in which "water trees" was observed (white pointers).

observed for any of the aged quercetin treated groups. Compared with the immediate groups, the silver uptake did not change dramatically after one-month collagenase aging.

\subsection{Contact angle evaluation}

The water contact angle and respective views are shown in Fig. 4. The average contact angle (mean $\pm \mathrm{SD}$ ) for the control, $0.1,0.5$, and $1.0 \mathrm{wt} \%$ groups were $31.9^{\circ} \pm 7.8^{\circ}, 49.0^{\circ} \pm 5.8^{\circ}$, $61.0^{\circ} \pm 4.0^{\circ}$ and $59.2^{\circ} \pm 2.7^{\circ}$, respectively. All three of the quercetin-treated groups showed higher water contact angles than the control group $(p<0.05)$.

\subsection{In situ zymography of the hybrid layer}

CLSM images of in situ zymography of quenched fluoresceinconjugated gelatin mixture are presented in Fig. 5. The control group (Fig. 5A) revealed intense green fluorescence in the hybrid layer and dentinal tubules. However, with increased quercetin concentrations, the intensity of the green fluorescence decreased gradually, and only some small dots of green fluorescence were observed in the $1.0 \mathrm{wt} \%$ group within the dentinal tubules while almost no MMP activity was seen in the hybrid layer (Fig. 5D).

\subsection{Antibacterial evaluation by CLSM and MTT assay}

Fig. 6 shows the stacked confocal images of live/dead bacteria in the $S$. mutans biofilms grown on the surfaces of different groups. The relative area distributions of the live/dead bacteria at each layer are summarized in the line plots under the corresponding confocal images. The confocal images revealed that the distribution of red fluorescence 


\section{Contact angle}

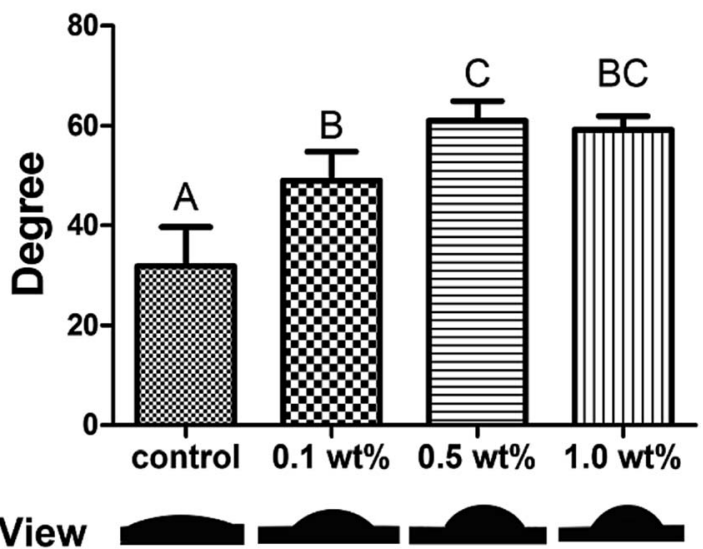

Fig. 4 The degree of water contact angle and respective views of the control and quercetin-treated groups on the demineralized dentin surfaces. Groups with the same letters are not statistically different ( $p$ > 0.05).
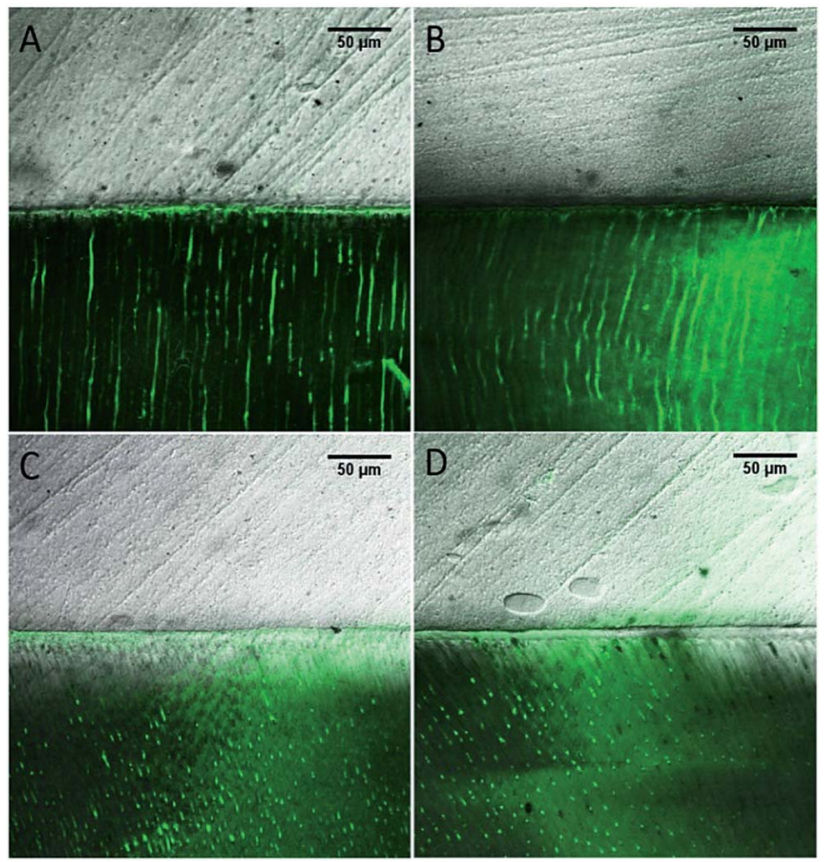

Fig. 5 CLSM images of in situ zymography of quenched fluoresceinconjugated gelatin mixture. ((A) The control group, (B) the $0.1 \mathrm{wt} \%$ group, (C) the 0.5 wt\% group, (D) the 1.0 wt\% group).

representing the percentage of dead bacteria, was much higher in the 0.5 and $1.0 \mathrm{wt} \%$ groups. The line plots displayed that pretreatment with 0.5 and $1.0 \mathrm{wt} \%$ groups resulted in a dramatic decrease in total biomass, which was approaching to zero at $10^{\text {th }}$ layer, representing the outstanding ability of inhibiting biofilm maturation. On the contrary, the control group manifested an upward curve with the total biomass still increasing at the $10^{\text {th }}$ layer, demonstrating no harmful effects on biofilm formation. The percentage distributions of
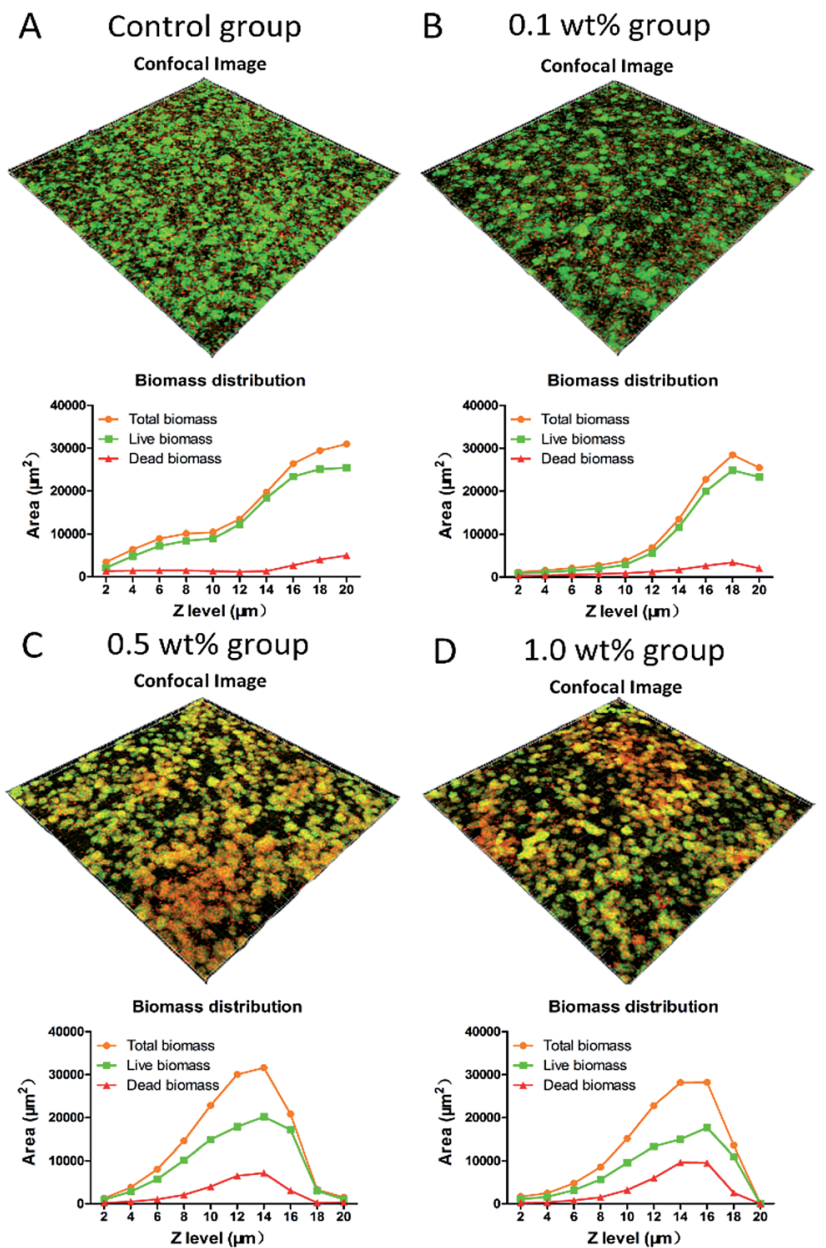

Fig. 6 CLSM images (2D overlay projections) of S. mutans biofilms (green-live; red-dead) after $24 \mathrm{~h}$ of incubation on dentin disks pretreated by different concentrations of quercetin. The line plot under each CLSM image indicates the corresponding biomass of the total, live, and dead bacteria ( $Z$ step $=2 \mu \mathrm{m}$ ).

live bacteria present in the 0.5 and $1.0 \mathrm{wt} \%$ groups were significantly less $(p<0.05)$ than that in the control group (Fig. 7A). The results of the MTT assay are shown in Fig. 7B. The bacteria derived from the 0.5 and $1.0 \mathrm{wt} \%$ quercetinpretreated groups showed significantly lower biological activity than that in the control group $(p<0.05)$.

\subsection{Cytotoxicity evaluation with MTT assay}

All of the quercetin solutions were tested on HDPs and evaluated for cytotoxicity by MTT assay (Fig. 8). The result showed that $400 \mu \mathrm{g} \mathrm{mL} \mathrm{m}^{-1}$ quercetin-containing medium had significantly decreased the viability of HDPs $(p<0.001)$, while the $200 \mu \mathrm{g} \mathrm{mL} \mathrm{m}^{-1}$ quercetin-containing medium turned out to be the maximum concentration which was on the edge of cytotoxicity $(p=0.012)$. The $12.5,25,50$ and $100 \mu \mathrm{g} \mathrm{mL}$ groups presented acceptable cytotoxicity compared with the control group $(p>0.05)$. This result indicated that quercetin solution had a dose-dependent cytotoxic effect on HDPs. 

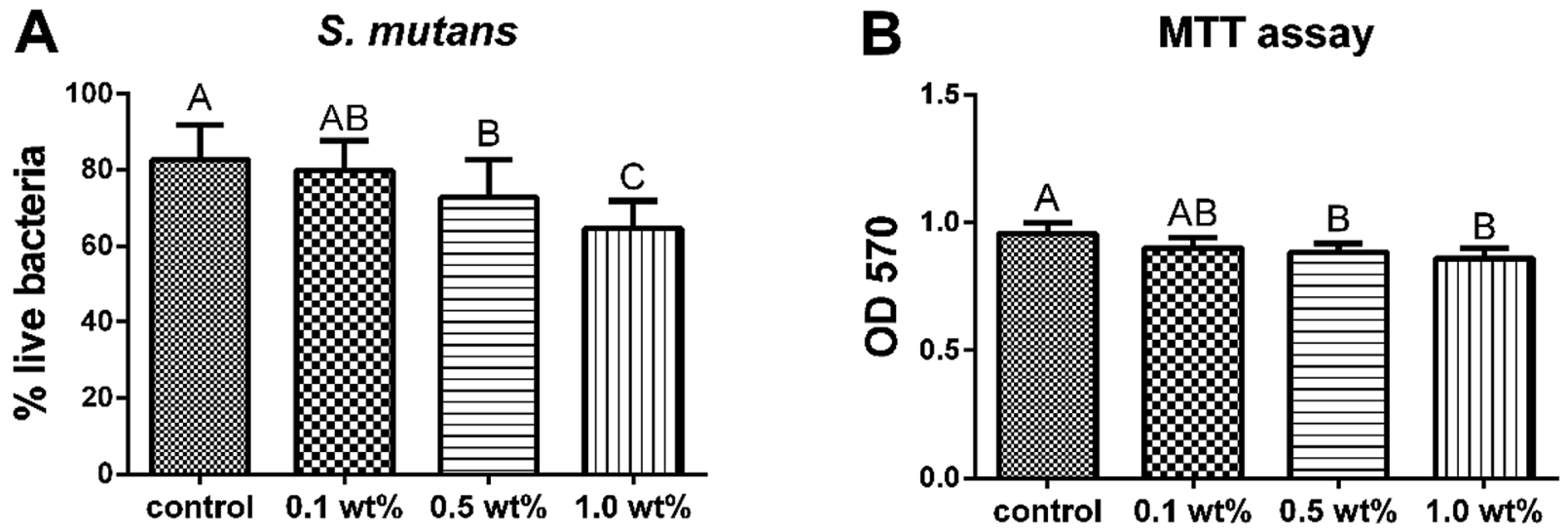

Fig. 7 Percentage distributions of live bacteria (A) and $\mathrm{OD}_{570}$ values (B) after $24 \mathrm{~h}$ incubation of $\mathrm{S}$. mutans on dentin disks pretreated by different concentrations of quercetin. Groups with the same letters are not statistically different $(p>0.05)$.

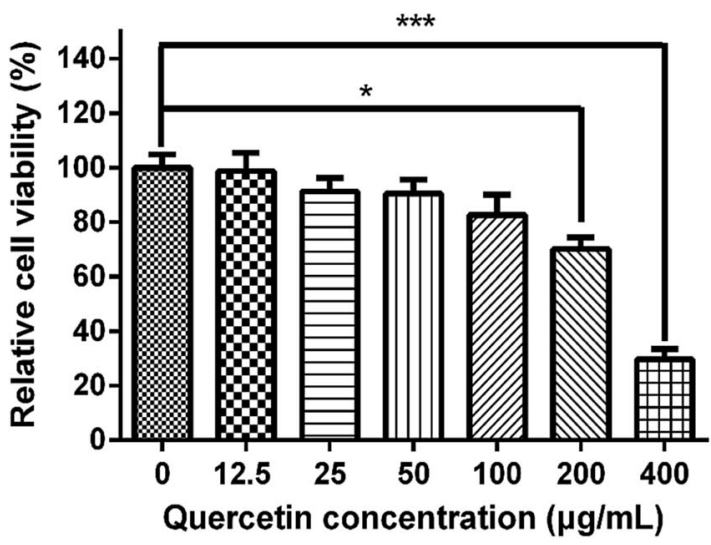

Fig. 8 Evaluation of HDPs viability exposed to different quercetincontaining mediums. The results were expressed as the mean $\pm S D, n$ $=5$. $* p<0.05$ as compared to control group. ${ }^{* * *} p<0.001$ as compared to control group.

\section{Discussion}

In our study, the multiple effects of quercetin/ethanol solution pretreatment on adhesive-dentin bonding were evaluated. The results showed that pretreatment with quercetin/ethanol solutions (especially at 0.5 and $1.0 \mathrm{wt} \%$ ) could preserve bonding performance against in vitro collagenase attack, inhibiting MMP activity and the growth of S. mutans. Therefore, the proposed hypotheses were rejected.

Degradation of the hybrid layer is considered the main factor causing weak bonding stability in adhesive restorations, and the enzymes in saliva can accelerate this process. ${ }^{36}$ If collagen in the hybrid layer is disintegrated by enzymes, the exposed resin matrix might be more susceptible to hydrolysis in storage solutions. ${ }^{37}$ It was reported that collagenase from C. histolyticum could attack collagen chains at several positions, thereby accelerating the degradation of collagen fibrils. ${ }^{38}$ Hence, bacterial collagenase was selected to challenge the adhesivedentin interface in the present study. Our study found that onemonth collagenase storage reduced the bonding strength of the control group (an approximately $31 \%$ reduction from a mean of 37.84 to 26.18) in the absence of quercetin pretreatment (Table 1).

Naturally derived quercetin cross-links collagen through four types of forces, namely hydrogen bonds, van der Waals forces, electrostatic forces, and hydrophobic forces. ${ }^{39}$ According to Haslam et al., polyphenols, such as quercetin, exhibit an amphiphilic property that combines the hydrophobic characteristic provided by its planar aromatic nucleus and the hydrophilic characteristic contributed by its polar hydroxyl groups. ${ }^{40}$ The hydrophobic forces of polyphenols drive them into the "holes" or "gap zones" in the collagen fiber, causing the former become embedded in the collagen structure. The hydroxyl group interacts with the proline residues of collagen through hydrogen bonding, providing a secondary interaction that helps to stabilize the complex. Similarly, Frazier and team proposed that the hydrophobic portion in aromatic rings could interact with the hydrophobic counterpart in other molecules and induce a p-stacking effect (van der Waals forces); while the hydrophilic portion is polarized and capable of hydrogen bonding. ${ }^{41}$ The MTBS results of the present study showed that pretreatment with quercetin/ethanol solution (0.5 and $1.0 \mathrm{wt} \%)$ could successfully preserve bonding strength (Table 1) even after collagenase attack while reducing the frequency of cohesive failure in dentin.

Nanoleakage is usually considered an important indicator of bonding stability and sealing capability because the nanozones or canals can act as pathways for water penetration and bacterial attack, leading to the failure of dentin bonding. Therefore, a general evaluation of nanoleakage along the bonding interface is necessary. ${ }^{30}$ After collagenase challenge (Fig. 3E and F), we found that in some area along the hybrid layer, the morphology of the metallic silver grains in the control group was similar to the "water trees" described by Tay ${ }^{35}$ Although "water trees" were initially reported by using transmission electron microscopy (TEM), they could still be observed by FESEM as presented perfectly by Li, Burrow and Tyas..$^{35,42}$ The "water trees" might be attributed to the degradation and dissolution of collagen attacked by collagenase, leading to the formation of some holes 
or spaces that allow for the ready infiltration of silver nitrite. However, in the quercetin-treated groups, "water trees" were not observed, while nanoleakage expression remarkably diminished compared with that in the control group. Two possible mechanisms might explain this phenomenon. Quercetin could cross-link with collagen to strengthen its stability and decrease the formation of water canals by resisting collagenase attack. On the other hand, quercetin could change the dynamics of water within the dentin matrix by interacting with collagen, increasing the hydrophobicity and generating changes in water content. ${ }^{43}$ It was clear in our contact angle test that quercetintreated groups showed significantly higher water contact angle than the control group ( $p<0.05$, Fig. 4), suggesting changes in water content. It was reported that lower water in dentin surface could facilitate the infiltration and polymerization of the adhesives, thereby increase the mechanical properties of the adhesive-dentin interfaces. ${ }^{44}$ Because the solvent used to dissolve quercetin in the present study was $100 \%$ ethanol, the quercetin/ethanol primer could be viewed as a combined application of collagen cross-linker and ethanolwet bonding technique, synergistically improve the bonding stability.

Unlike the result of MTBS, the immediate nanoleakage expression of the four experimental groups was significantly different. This result might provide support to Hashimoto and Yang, whose researches found no direct relationship between the values of resin-dentin bond strength and the percent of nanoleakage.$^{30,45}$ In contrast, Ekambaram et al. found that, with the increase in nanoleakage, the resin-dentin bond strength decreased to some degree. ${ }^{46}$ Therefore, the relationship between resin-dentin bond strength and nanoleakage expression is complex and controversial, which needs to be further discovered.

Besides the exogenous enzymes like bacterial collagenase, the endogenous enzymes like MMPs also play an important role to degrade dentin collagen. Vijayababu and coworkers found that quercetin was an inhibitor of MMP-2 and MMP-9, which exist in dentin that could be exposed and activated by acidetching. ${ }^{24,47}$ Therefore, we evaluated the MMP inhibition ability of quercetin using in situ zymograph test. Intense green fluorescence was found in the hybrid layer and dentinal tubules of the control group, indicating that the fluorescein-conjugated gelatin mixture was strongly degraded by local MMPs at these sites. Using of quercetin, an apparent decrease in the intensity of green fluorescence (identifying intense MMP activity) was observed from the control group to the $1.0 \mathrm{wt} \%$ quercetintreated group, indicating that quercetin inhibited the activity of MMP within the hybrid layer. Theoretically, this phenomenon could be attributed to the conformational changes in the enzyme 3D structure by altering the catalytic domain or other modular domains that co-participate in collagen degradation. ${ }^{8}$

Its cross-linking effects, hydrophobic characteristics and inhibition of MMP make quercetin a promising primer for maintaining dentin bonding strength and hybrid layer integrity. However, under complex intraoral conditions, a considerable number of cariogenic bacteria could accumulate on the resinbased restorations and cause secondary caries. $^{48}$
Simultaneously, the collagenase excreted by bacteria could induce the degradation of collagen and expedite the aging process. $^{38}$ Therefore, an ideal material for dentin bonding should possess antibacterial ability as well.

Biofilm growth is an essential factor in caries development. Among the cariogenic bacteria, S. mutans is believed to play an important role in the initial progression of dental caries because it can biodegrade carbohydrates to produce acid, excrete extracellular polysaccharide, adhere to the tooth surface, and form biofilms. ${ }^{49}$ The Mirzoeva group showed that quercetin could increase the permeability of the inner bacterial membrane and change the membrane potential. ${ }^{50}$ The change in membrane potential could hinder the ability of bacteria to synthesize ATP and transport material through the membrane, thereby leading to death. Furthermore, Mirzoeva suggested that the effect of impeding motility might inhibit bacterial pathogenesis and initial biofilm development. Our previous study proved that quercetin could effectively inhibit GTF B and C expression and consequently decrease the virulence of $S$. mutans in the pathogenesis of dental caries. ${ }^{51}$ GTF B and C play significant roles in biofilm development because most of the glucans synthesized by these enzymes are retained on the acquired pellicle. This retention is a prerequisite for the accumulation of $S$. mutans and other cariogenic bacteria on the tooth surface. ${ }^{52}$ In the present study, in order to provide more powerful evidences, we have selected a series of quercetin/ ethanol solutions (namely $0.01 \mathrm{wt} \%, 0.05 \mathrm{wt} \%, 0.1 \mathrm{wt} \%, 0.5$ $\mathrm{wt} \%$ and $1.0 \mathrm{wt} \%$ ) to evaluate their antibacterial ability, and the results can be found as ESI Fig. S1-S3† online. From the results, we found that the $0.01 \mathrm{wt} \%$ and $0.05 \mathrm{wt} \%$ groups manifested no significant antibacterial ability compared with the control group, while the $0.5 \mathrm{wt} \%$ and $1.0 \mathrm{wt} \%$ groups exhibited outstanding bactericidal ability and could inhibit the biofilm growth of $S$. mutans. Considering the paper length and the readability, the three representative concentrations were adopted in the main manuscript, namely, the $0.1 \mathrm{wt} \%, 0.5 \mathrm{wt} \%$ and $1.0 \mathrm{wt} \%$ groups, and the related results are presented in Fig. 6 and 7.

Being a natural cross-linker, quercetin has been widely accepted for its biocompatibility and safety; a review even suggested that daily consumption of quercetin at dietary intake levels (200-500 mg day ${ }^{-1}$ ) would not produce adverse health effects. ${ }^{53,54}$ However, according to the MTT results (Fig. 8), the concentration of our selected quercetin/ethanol solutions far exceeded $400 \mu \mathrm{g} \mathrm{mL} \mathrm{m}^{-1}$, which may imply their potential cytotoxicity. Considering the actual situation in vivo, we cannot simply draw this conclusion. Because in the in vivo application, the dentinal tubules have an outward pressure due to the outflow of dentinal fluid. ${ }^{55}$ It would be difficult for quercetin to penetrate the long dentinal tubules, exerting cytotoxic effects on dental pulp cells. As a result, we could not exactly measure the accurate proportion of infiltrated-quercetin compared with the total amount. Furthermore, when used in dentin bonding, only one or two drops of quercetin/ethanol solution were brushed on the dentin surface. We believe that this tiny amount of solution would not display significant cytotoxicity. Therefore, the MTT assay cautioned us that we should not neglect the cytotoxicity of 
quercetin, but whether the $0.5 \mathrm{wt} \%$ or $1.0 \mathrm{wt} \%$ quercetin/ ethanol solutions were cytotoxic requires further in vivo evaluation.

Due to the versatile performance of quercetin, we believe that it has great potential in dental application. However, there are two issues to which we must pay attention. First, as an antioxidant, the high concentration of quercetin might possibly inhibit the polymerization of the adhesive and decrease the bonding strength just like PA and EGCG do. ${ }^{14,56}$ In our experiments, the immediate MTBS did not decrease with $1.0 \mathrm{wt} \%$ quercetin/ethanol solution pretreatment, perhaps because of its application method with no direct addition into dental adhesive. Second, the leaching of most antibacterial materials has a dual-phase release process, namely the burst-release phase in the first few days and tail-release phase after several weeks. ${ }^{57}$ In the burst-release phase, a high concentration of antimicrobials is released, while in the tail-release phase, the concentration is too low to be effective. Regarding quercetin, we speculate that it could exert long-term anti-bacterial ability, not only due to its cross-linking effect with dentin collagen, but also its barely soluble character, limiting its leaching rate into saliva. Therefore, further researches are needed to explore the possibility of adding quercetin into dental adhesives and to evaluate the antibacterial ability of adhesive/dentin interface after long-term function.

\section{Conclusions}

Pretreatment with 0.5 and $1.0 \mathrm{wt} \%$ quercetin/ethanol solutions for $60 \mathrm{~s}$ was effective in preserving the bond strength and integrity of the hybrid layer after one-month bacterial collagenase aging. In addition, the 0.5 and $1.0 \mathrm{wt} \%$ quercetinpretreated specimens demonstrated favorable ability in killing $S$. mutans and inhibiting the biofilm growth. Therefore, quercetin/ethanol solution (especially $0.5 \mathrm{wt} \%$ and $1.0 \mathrm{wt} \%$ ) has the potential to serve as a simple and versatile primer to provide dentists a promising approach to obtain desirable adhesivedentin bonds and offer secondary caries prevention, thereby preventing the frequent replacement of resin-based restorations.

\section{Acknowledgements}

This work was financially supported by National Nature Science Foundation of China (No. 81371191).

\section{Notes and references}

1 M. G. Buonocore, J. Dent. Res., 1955, 34, 849-853.

2 M. G. Brackett, N. Li, W. W. Brackett, R. J. Sword, Y. P. Qi, L. N. Niu, C. R. Pucci, A. Dib, D. H. Pashley and F. R. Tay, J. Dent., 2011, 39, 238-248.

3 N. Nakabayashi, K. Kojima and E. Masuhara, J. Biomed. Mater. Res., Part A, 1982, 16, 265-273.

4 V. Hass, I. V. Luque-Martinez, M. F. Gutierrez, C. G. Moreira, V. B. Gotti, V. P. Feitosa, G. Koller, M. F. Otuki, A. D. Loguercio and A. Reis, Dent. Mater., 2016, 32, 732-741.
5 A. J. van Strijp, D. C. Jansen, J. DeGroot, J. M. ten Cate and V. Everts, Caries Res., 2003, 37, 58-65.

6 F. R. Tay, M. Hashimoto, D. H. Pashley, M. C. Peters, S. C. Lai, C. K. Yiu and C. Cheong, J. Dent. Res., 2003, 82, 537-541.

7 M. Ekambaram, C. K. Yiu, J. P. Matinlinna, N. M. King and F. R. Tay, J. Dent., 2014, 42, 709-719.

8 Y. Liu, L. Tjaderhane, L. Breschi, A. Mazzoni, N. Li, J. Mao, D. H. Pashley and F. R. Tay, J. Dent. Res., 2011, 90, 953-968.

9 D. T. Cheung, D. Tong, N. Perelman, D. Ertl and M. E. Nimni, Connect. Tissue Res., 1990, 25, 27-34.

10 A. Mazzoni, V. Angeloni, F. M. Apolonio, N. Scotti, L. Tjaderhane, A. Tezvergil-Mutluay, R. Di Lenarda, F. R. Tay, D. H. Pashley and L. Breschi, Dent. Mater., 2013, 29, 1040-1047.

11 L. J. Southern, H. Hughes, P. V. Lawford, M. R. Clench and N. J. Manning, J. Heart Valve Dis., 2000, 9, 241-248.

12 N. Hiraishi, R. Sono, I. Sofiqul, C. Yiu, H. Nakamura, M. Otsuki, T. Takatsuka and J. Tagami, Dent. Mater., 2013, 29, 1048-1054.

13 J. Hu, X. Du, C. Huang, D. Fu, X. Ouyang and Y. Wang, J. Dent., 2013, 41, 927-934.

14 D. J. Epasinghe, C. K. Yiu, M. F. Burrow, F. R. Tay and N. M. King, J. Dent., 2012, 40, 173-180.

15 Y. Liu, M. Chen, X. Yao, C. Xu, Y. Zhang and Y. Wang, Dent. Mater., 2013, 29, 485-492.

16 V. Hass, I. Luque-Martinez, M. A. Muñoz, M. F. G. Reyes, G. Abuna, M. A. C. Sinhoreti, A. Y. Liu, A. D. Loguercio, Y. Wang and A. Reis, Dent. Mater., 2016, 32, 468-475.

17 R. Walter, P. A. Miguez, R. R. Arnold, P. N. Pereira, W. R. Duarte and M. Yamauchi, Caries Res., 2008, 42, 263268.

18 D. P. Leitao, A. C. Polizello, I. Y. Ito and A. C. Spadaro, J. Med. Food, 2005, 8, 36-40.

19 B. Hechler, X. Yao and Y. Wang, Am. J. Dent., 2012, 25, 276. 20 I. Erlund, Nutr. Res., 2004, 24, 851-874.

21 D. R. Ferry, A. Smith, J. Malkhandi, D. W. Fyfe, P. G. deTakats, D. Anderson, J. Baker and D. J. Kerr, Clin. Cancer Res., 1996, 2, 659-668.

22 M. Chopra, P. E. Fitzsimons, J. J. Strain, D. I. Thurnham and A. N. Howard, Clin. Chem., 2000, 46, 1162-1170.

23 M. A. Pereira, C. J. Grubbs, L. H. Barnes, H. Li, G. R. Olson, I. Eto, M. Juliana, L. M. Whitaker, G. J. Kelloff and V. E. Steele, Carcinogenesis, 1996, 17, 1305-1311.

24 M. R. Vijayababu, A. Arunkumar, P. Kanagaraj, P. Venkataraman, G. Krishnamoorthy and J. Arunakaran, Mol. Cell. Biochem., 2006, 287, 109-116.

25 W. Zhai, X. Lu, J. Chang, Y. Zhou and H. Zhang, Acta Biomater., 2010, 6, 389-395.

26 D. Rigano, C. Formisano, A. Basile, A. Lavitola, F. Senatore, S. Rosselli and M. Bruno, Phytother. Res., 2007, 21, 395-397. 27 T. N. Kaul, E. Middleton Jr and P. L. Ogra, J. Med. Virol., 1985, 15, 71-79.

28 D. Althans, P. Schrader, S. Enders and D. Althans, J. Mol. Liq., 2014, 196, 86-93.

29 C. Mcknighthanes and G. M. Whitford, Caries Res., 1992, 26, 345-350. 
30 H. Yang, J. Guo, J. Guo, H. Chen, M. Somar, J. Yue and C. Huang, Dent. Mater. J., 2015, 34, 654-662.

31 V. P. Saboia, F. Nato, A. Mazzoni, G. Orsini, A. Putignano, M. Giannini and L. Breschi, J. Adhes. Dent., 2008, 10, 419422.

32 L. Boulos, M. Prevost, B. Barbeau, J. Coallier and R. Desjardins, J. Microbiol. Methods, 1999, 37, 77-86.

33 H. Wang, H. Cheng, F. Wang, D. Wei and X. Wang, J. Microbiol. Methods, 2010, 82, 330-333.

34 L. Zou, M. R. Harkey and G. L. Henderson, Phytomedicine, 2002, 9, 263-267.

35 F. R. Tay, D. H. Pashley, B. I. Suh, N. Hiraishi and C. K. Yiu, Operat. Dent., 2005, 30, 561-579.

36 Y. Finer and J. P. Santerre, J. Dent. Res., 2004, 83, 22-26.

37 Y. Zou, J. L. Jessop and S. R. Armstrong, J. Biomed. Mater. Res., Part A, 2010, 94, 187-192.

38 S. P. Ho, R. M. Sulyanto, S. J. Marshall and G. W. Marshall, J. Struct. Biol., 2005, 151, 69-78.

39 X. Yang, D. Wu, Z. Du, R. Li, X. Chen and X. Li, J. Agric. Food Chem., 2009, 57, 3431-3435.

40 E. Haslam, J. Nat. Prod., 1996, 59, 205-215.

41 R. A. Frazier, E. R. Deaville, R. J. Green, E. Stringano, I. Willoughby, J. Plant and I. Mueller-Harvey, J. Pharm. Biomed. Anal., 2010, 51, 490-495.

42 H. Li, M. F. Burrow and M. J. Tyas, J. Adhes. Dent., 2003, 5, 19-25.

43 N. N. Fathima, M. Baias, B. Blumich and T. Ramasami, Int. J. Biol. Macromol., 2010, 47, 590-596.

44 A. A. Leme, C. M. Vidal, L. S. Hassan and A. K. Bedran-Russo, J. Biomech., 2015, 48, 2067-2071.
45 M. Hashimoto, J. De Munck, S. Ito, H. Sano, M. Kaga, H. Oguchi, B. Van Meerbeek and D. H. Pashley, Biomaterials, 2004, 25, 5565-5574.

46 M. Ekambaram, C. K. Yiu, J. P. Matinlinna, J. W. Chang, F. R. Tay and N. M. King, J. Dent., 2014, 42, 872-882.

47 A. Mazzoni, F. D. Nascimento, M. Carrilho, I. Tersariol, V. Papa, L. Tjaderhane, R. Di Lenarda, F. R. Tay, D. H. Pashley and L. Breschi, J. Dent. Res., 2012, 91, 467-472.

48 R. Walter, W. R. Duarte, P. N. Pereira, H. O. Heymann, E. J. Swift Jr and R. R. Arnold, Oper. Dent., 2007, 32, 388-393.

49 P. Chong, L. Drake and I. Biswas, J. Bacteriol., 2008, 190, 4478-4488.

50 O. K. Mirzoeva, R. N. Grishanin and P. C. Calder, Microbiol. Res., 1997, 152, 239-246.

51 J. X. Yue, H. Y. Yang, L. Han, M. Y. Zhu, F. F. Song and C. Huang, Zhonghua Kouqiang Yixue Zazhi, 2016, 51, 368373.

52 K. M. Schilling and W. H. Bowen, Infect. Immun., 1992, 60, 284-295.

53 M. J. Ruiz, M. Fernández, J. M. Estela, M. Á. Asensi, J. Mañes and Y. Picó, Toxicol. Lett., 2006, 164, S275-S276.

54 M. Harwood, B. Danielewskanikiel, J. F. Borzelleca, G. W. Flamm, G. M. Williams and T. C. Lines, Food Chem. Toxicol., 2007, 45, 2179-2205.

55 B. Ciucchi, S. Bouillaguet, J. Holz and D. Pashley, J. Endod., 1995, 21, 191-194.

56 X. Du, X. Huang, C. Huang, Y. Wang and Y. Zhang, J. Dent., 2012, 40, 485-492.

57 S. Q. Gong, J. Epasinghe, F. A. Rueggeberg, L. N. Niu, D. Mettenberg, C. K. Yiu, J. D. Blizzard, C. D. Wu, J. Mao and C. L. Drisko, PLoS One, 2012, 7, e42355. 